\title{
Rectilinear Block Placement Using B*-Trees
}

\author{
GUANG-MING WU \\ Nan-Hua University \\ YUN-CHIH CHANG \\ Realtek Semiconductor Corp. \\ and \\ YAO-WEN CHANG \\ National Taiwan University
}

\begin{abstract}
Due to the layout complexity in modern VLSI designs, integrated circuit blocks may not be rectangular. However, literature on general rectilinear block placement is still quite limited. In this article, we present approaches for handling the placement for arbitrarily shaped rectilinear blocks using $\mathrm{B}^{*}$-trees [Chang et al. 2000]. We derive the feasibility conditions of $\mathrm{B}^{*}$-trees to guide the placement of rectilinear blocks. Experimental results show that our algorithm achieves optimal or near-optimal block placement for benchmarks with various shaped blocks.
\end{abstract}

Categories and Subject Descriptors: B7.2 [Integrated Circuits]: Design Aids-placement and routing; J.6 [Computer Applications]: Computer-Aided Engineering

General Terms: Algorithms, Design, Experimentation, Measurement, Performance

Additional Key Words and Phrases: Computer-aided design of VLSI, floorplanning, layout, placement

\section{INTRODUCTION}

Due to the growth in design complexity, circuit size is getting larger. To cope with the increasing design complexity, hierarchical design and IP modules are widely used. This trend makes block floorplanning/placement much more critical to the quality of a design.

Floorplanning is often studied based on two floorplan structures, the slicing structure [Otten 1982; Wong and Liu 1986] and the nonslicing structure [Chang

The work of G.-M. Wu was partially supported by the National Science Council of Taiwan ROC under Grant No. NSC-91-2215-E-343-001. The work of Y.-W. Chang was partially supported by the National Science Council of Taiwan ROC under Grant No. NSC-91-2215-E-002-038.

Authors' addresses: G.-M. Wu, Department of Information Management, Nan-Hua University, Chiayi, Taiwan; email: gmwu@mail.nhu.edu.tw; Y.-C. Chang, Realtek Semiconductor Corp., No. 2, Industry E. Rd. IX, Science-Based Industrial Park, Hsinchu, Taiwan; Y.-W. Chang, Graduate Institute of Electronics Engineering and Department of Electrical Engineering, National Taiwan University, Rm. 548, Electrical Engineering Building \#1, Taipei 106, Taiwan; email: ywchang@ cc.ee.ntu.edu.tw.

Permission to make digital/hard copy of part or all of this work for personal or classroom use is granted without fee provided that the copies are not made or distributed for profit or commercial advantage, the copyright notice, the title of the publication, and its date appear, and notice is given that copying is by permission of the ACM, Inc. To copy otherwise, to republish, to post on servers, or to redistribute to lists, requires prior specific permission and/or a fee.

(C) 2003 ACM 1084-4309/03/0400-0188 $\$ 5.00$

ACM Transactions on Design Automation of Electronic Systems, Vol. 8, No. 2, April 2003, Pages 188-202. 
et al. 2000; Guo et al. 1999; Murata et al. 1997; Nakatake et al. 1996; Wang and Wong 1990]. A slicing structure can be represented by a binary tree whose leaves denote modules, and internal nodes specify horizontal or vertical cut lines. Wong and Liu [1986] proposed an algorithm for slicing floorplan design. They presented a normalized Polish expression to represent a slicing structure, enabling the speedup of the search procedure. However, this representation cannot handle nonslicing floorplans. Recently, researchers have proposed several representations for nonslicing floorplans, such as the sequence pair [Murata et al. 1997], bounded slicing grid (BSG) [Nakatake et al. 1996], O-tree [Guo et al. 1999], and B*-tree [Chang et al. 2000].

In modern VLSI design, the blocks may not be rectangular. Most existing floorplanning/placement algorithms, however, only deal with rectangles and cannot apply to arbitrarily shaped rectilinear block placement directly. New approaches that can handle arbitrarily shaped blocks are essential to optimize resource (e.g., area) utilization.

Preas and van Cleemput [1979] proposed a graph model for the topological relationships among rectangular and arbitrarily shaped rectilinear blocks. Wong and Liu [1987] extended the Polish expression to represent slicing floorplans with rectangular and L-shaped blocks. Lee [1993] extended the zone refinement technique to rectilinear blocks. A bounded 2-D contour searching algorithm was proposed to find the best position for a block.

Kang and Dai [1997] proposed a BSG-based method to solve the packing of rectangular, L-shaped, T-shaped, and soft blocks. The algorithm combined simulated annealing and a genetic algorithm for general nonslicing floorplans.

Xu et al. [1998] presented an approach extending the sequence-pair approach for rectangular block placement to arbitrarily sized and shaped rectilinear blocks. The properties of L-shaped blocks were examined first, and then arbitrarily shaped rectilinear blocks were decomposed into a set of L-shaped blocks.

Kang and Dai [1998] proposed a method based on the sequence-pair structure for rectilinear block placement. Three necessary and sufficient conditions for a sequence pair to be feasible were derived. A stochastic search was applied on the optimization of convex block floorplanning.

Chang et al. [2000] recently proposed the $B^{*}$-tree representation for nonslicing floorplans, which is based on block compaction and ordered binary trees. Inheriting from the nice properties of ordered binary trees, $\mathrm{B}^{*}$-trees are very easy to implement and require only constant time for tree search and insertion, and linear time for deletion. Unlike the O-tree representations, in particular, no extra encoding of the tree itself is needed for a $\mathrm{B}^{*}$-tree, and cost evaluation can be performed directly on a $\mathrm{B}^{*}$-tree. Besides, the ordered property of a $\mathrm{B}^{*}$-tree makes the incremental cost evaluation of its corresponding placement possible. Furthermore, given a $\mathrm{B}^{*}$-tree, it takes only linear time to construct the placement, and vice versa. All these nice properties make the $\mathrm{B}^{*}$-trees an efficient and flexible representation for nonslicing floorplans.

In this article, we apply the B*-tree to handle the placement of arbitrarily shaped rectilinear blocks. First, we explore the properties of L-shaped blocks and then extend the properties to general rectilinear blocks. The feasibility conditions are then used to guide the placement of rectilinear blocks. We construct a 


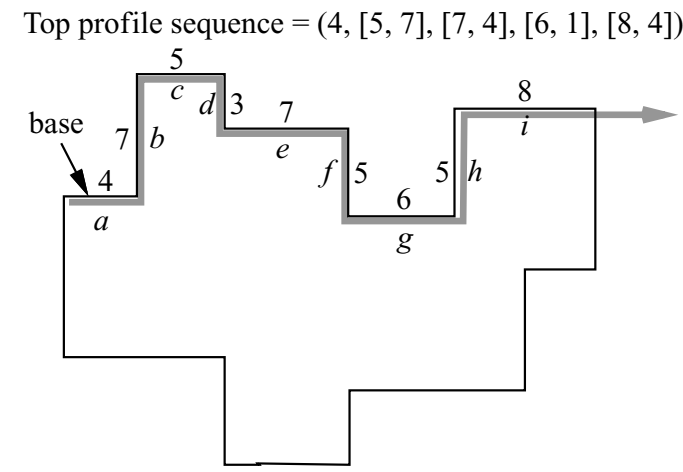

Fig. 1. The top profile sequence consists of the length of the base, followed by a sequence of twotuples that is composed of the lengths of the succeeding horizontal segments and their relative heights to the base.

set of benchmarks with rectangular and L-shaped (and T-shaped) blocks and apply simulated annealing as a vehicle to test the effectiveness of our approaches. Experiment results show that our approaches lead to placements with optimal or near-optimal area utilization.

The remainder of this article is organized as follows. Section 2 formulates the rectilinear block placement problem. Section 3 introduces the $\mathrm{B}^{*}$-tree representation. Section 4 describes the method for the L-shaped blocks in a B*-tree. Section 5 describes our algorithm. Section 6 extends the algorithm to arbitrarily rectilinear blocks. Experimental results are reported in Section 7. Finally, we give conclusions in Section 8.

\section{FORMULATION}

Let $B=\left\{b_{1}, b_{2}, \ldots, b_{n}\right\}$ denote a set of rectilinear blocks. A block is not flexible in its shape but free to rotate and flip. A packing of a set of blocks is a nonoverlapping placement of the blocks.

A rectilinear block can be represented by four profile sequences, namely, the top profile sequence, the bottom profile sequence, the left profile sequence, and the right profile sequence, specifying the profiles viewed from the top side, the bottom side, the left side, and the right side of the block, respectively. The top (bottom) profile sequence of a rectilinear block uses the leftmost horizontal segment on the top (bottom) boundary of the block as a base and records the length of the succeeding horizontal segments on the top (bottom) boundary and the relative height. Specifically, the top profile sequence consists of the length of the base, followed by a sequence of two-tuples composed of the lengths of the succeeding horizontal segments and their relative heights to the base (could be negative). For example, Figure 1 shows a rectilinear block with the top profile sequence $(4,[5,7],[7,4],[6,-1],[8,4])$. The base of the sequence is segment $a$ which has a length of 4 units. The second horizontal segment is $c$ which has a length of 5 units and is 7 units higher than the base $a$. Similarly, the third horizontal segment is $e$ which has a length of 7 units and is 4 units higher 


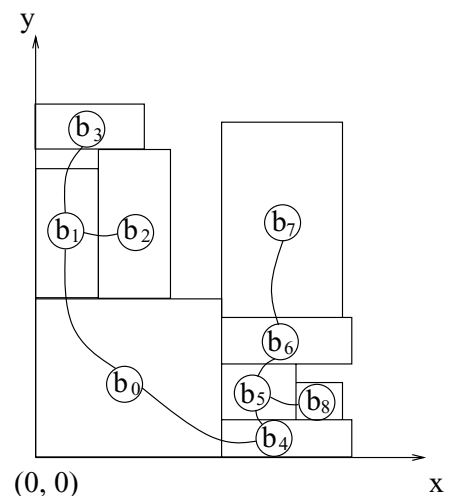

(a)

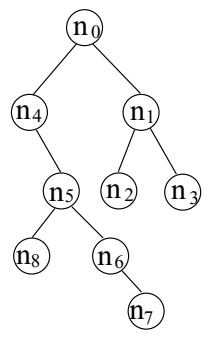

(b)

Fig. 2. An admissible placement and its corresponding $B^{*}$-tree.

than the base $a$, and so on. The other three profile sequences are similarly defined.

Definition 1. A rectilinear block placement is feasible if and only if no two blocks overlap each other, and all profile sequences remain unchanged after placement (i.e., all blocks keep their original shapes).

The goal of the rectilinear placement problem is to optimize a given cost metric (such as area, wirelength, etc.) induced by the assignment of $b_{i}$ s. (By area here, we mean the final enclosing rectangle of $B$.)

\section{OVERVIEW OF THE B*-TREE REPRESENTATION}

Given an admissible placement $P$, we can represent it by a unique (horizontal) $\mathrm{B}^{*}$-tree $T$ [Chang et al. 2000]. (See Figure 2(b) for the $\mathrm{B}^{*}$-tree representing the placement shown in Figure 2(a).) A B*-tree is an ordered binary tree whose root corresponds to the module in the bottom-left corner. Similar to the depthfirst search (DFS) procedure, we construct the $\mathrm{B}^{*}$-tree $T$ for an admissible placement $P$ in a recursive fashion. Starting from the root, we first recursively construct the left subtree and then the right subtree. Let $R_{i}$ denote the set of modules located on the right-hand side and adjacent to $b_{i}$. The left child of the node $n_{i}$ corresponds to the lowest module in $R_{i}$ that is unvisited. The right child of $n_{i}$ represents the first module located above and visible from $b_{i}$, with its $x$-coordinate equal to that of $b_{i}$ (and its $y$-coordinate less than or equal to that of the top boundary of the module on the left-hand side and adjacent to $b_{i}$, if any, to consider the special placement).

As shown in Figure 2, we make $n_{0}$ the root of $T$ since $b_{0}$ is in the bottom-left corner. Constructing the left subtree of $n_{0}$ recursively, we make $n_{4}$ the left child of $n_{0}$. Since the left child of $n_{4}$ does not exist, we then construct the right subtree of $n_{4}$ (which is rooted by $n_{5}$ ). The construction is recursively performed in the DFS order. After completing the left subtree of $n_{0}$, the same procedure applies to the right subtree of $n_{0}$. Figure 2(b) illustrates the resulting $\mathrm{B}^{*}$-tree for the placement shown in Figure 2(a). The construction takes only linear time. 


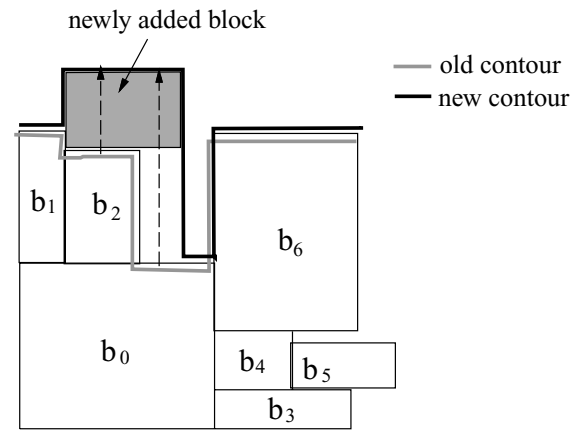

Fig. 3. A contour and its update: when adding a new block to the placement, we search the contour from left to right and update it with the top boundary of the new block.

The $\mathrm{B}^{*}$-tree keeps the geometric relationship between two modules as follows. If node $n_{j}$ is the left child of node $n_{i}$, module $b_{j}$ must be located on the right-hand side and adjacent to module $b_{i}$ in the admissible placement; that is, $x_{j}=x_{i}+w_{i}$. Besides, if node $n_{j}$ is the right child of $n_{i}$, module $b_{j}$ must be located above and visible from module $b_{i}$, with the $x$-coordinate of $b_{j}$ equal to that of $b_{i}$; that is, $x_{j}=x_{i}$. Also, since the root of $T$ represents the bottomleft module, the $x$ - and $y$-coordinates of the module associated with the root $\left(x_{\text {root }}, y_{\text {root }}\right)=(0,0)$.

A contour structure (see Figure 3), which was originally proposed in Guo et al. [1999], can be used to reduce the run-time of finding the $y$-coordinate of a newly inserted block. The contour structure is a doubly linked list of blocks, which describes the contour line in the current compaction direction. Without the contour structure, the run-time for placing a new block is linear to the number of blocks. By maintaining the contour structure, however, the $y$-coordinate of a block can be computed in $O(1)$ time. Figure 3 illustrates how to update the contour when we add a new block to the placement.

\section{L-SHAPED BLOCKS}

In this section, we apply the $\mathrm{B}^{*}$-tree approach to find a feasible placement with L-shaped blocks. Let $b_{L}$ denote an L-shaped block. $b_{L}$ can be partitioned into two rectangular subblocks by slicing $b_{L}$ along its middle vertical boundary. As shown in Figure 4(a), $b_{1}$ and $b_{2}$ are the subblocks of $b_{L}$, and we say $b_{1}, b_{2} \in b_{L}$.

After partitioning and placement, the rectilinear block $b_{L}$ might not conform to its top profile sequence, as illustrated in Figure 5. Figure 5(a) shows a B*tree and its corresponding placement. We can pull subblock $b_{2}$ up to align with the subblock $b_{1}$, so that the block $b_{L}$ can maintain its top profile sequence without changing the overall topology of the blocks. Conversely, there might not be enough space to do so; see Figure 5(b) for such an example. It is obvious that a feasible placement can be generated from the $\mathrm{B}^{*}$-tree shown in Figure 5(a) with a local adjustment, but it is impossible for the case shown in Figure 5(b). Therefore, if we represent an L-shaped block by two subblocks, we must guarantee that the two subblocks abut. To ensure that the left subblock $b_{1}$ and the 


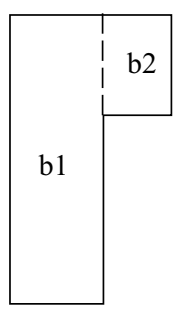

(a)

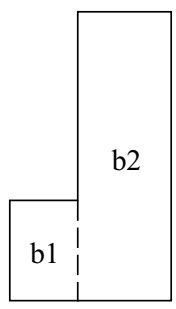

(e)

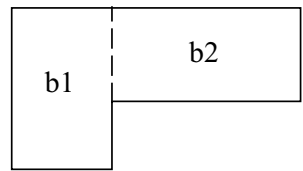

(b)

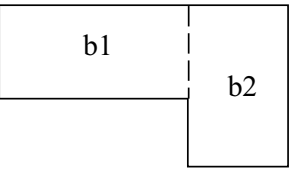

(f)

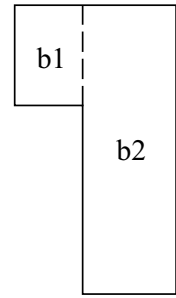

(c)

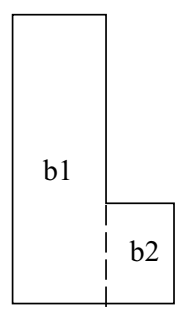

(g)

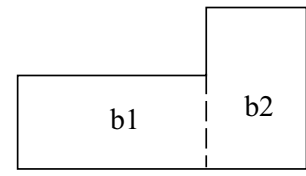

(d)

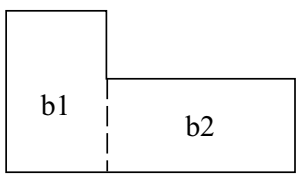

(h)

Fig. 4. Eight situations of an L-shaped block. Each is partitioned into two parts by slicing it along the middle vertical boundary.

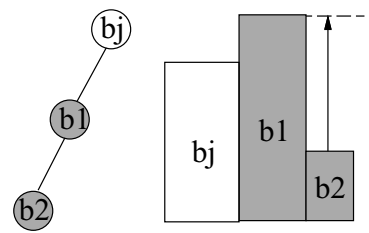

(a)

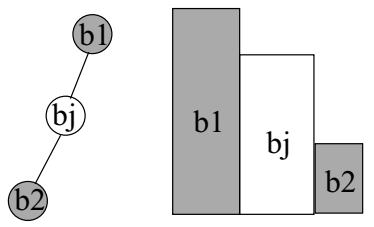

(b)

Fig. 5. Placing the L-shaped block shown in Figure 4(a) by two subblocks: (a) a feasible placement; (b) an infeasible placement.

right subblock $b_{2}$ of an L-shaped block $b_{L}$ abut, we impose the following location constraint ( $L C$ for short) for $b_{1}$ and $b_{2}$.

$L C$ : Keep $b_{2}$ as $b_{1}$ 's left child in the $\mathrm{B}^{*}$-tree.

The $L C$ relation ensures that the $x$-coordinate of the left boundary of $b_{2}$ is equal to that of the right boundary of $b_{1}$. For example, the two sets of subblocks $b_{1}, b_{2}$ and $b_{3}, b_{4}$ shown in Figure 6(a) do not abut whereas those shown in Figure 6(b) 


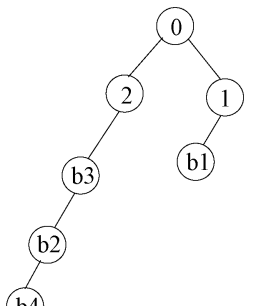

(b4)

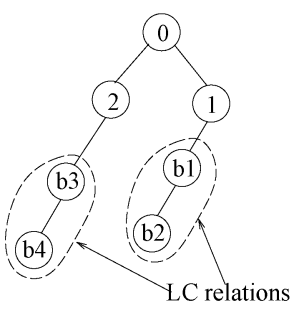

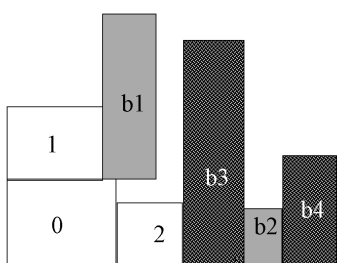

(a)

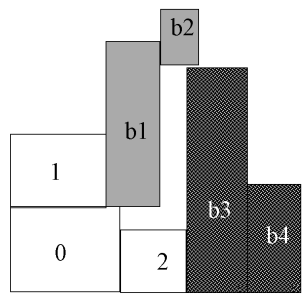

(b)

Fig. 6. Suppose that $b_{1}, b_{2}$ and $b_{3}, b_{4}$ are two sets of subblocks corresponding to two L-shaped blocks (a) a placement in which $b_{1}, b_{2}$ and $b_{3}, b_{4}$ do not abut. Their corresponding nodes in the $\mathrm{B}^{*}$ tree may not be related; (b) another placement in which $b_{1}, b_{2}$ and $b_{3}, b_{4}$ abut. Their corresponding nodes in the $\mathrm{B}^{*}$-tree keep the $L C$ relation between $b_{1}$ and $b_{2}$ (as well as $b_{3}$ and $b_{4}$ ).

do. In Figure 6(b), the subblocks $b_{3}$ and $b_{4}$ are placed at the right locations and the subblocks $b_{1}$ and $b_{2}$ are not since the $y$-coordinates of $b_{1}$ and $b_{2}$ are not equal. We say $b_{1}$ and $b_{2}$ are misaligned.

In the following, we adopt the contour data structure to solve the misalignment problem. When transforming a $\mathrm{B}^{*}$-tree to its corresponding placement, we update the contour to maintain its top profile sequence as follows. Assume that $b_{1}$ and $b_{2}$ are the respective left and right subblocks of an L-shaped block $b_{L}$, and they are misaligned. When processing $b_{2}, b_{1}$ must have been placed. We can classify the misalignment into categories and adjust them as follows.

(1) Basin: The contour is lower than the top profile sequence at the position of the current subblock $b_{2}$. (See Figure 7(a).) In this case, we pull $b_{2}$ up to conform to the top profile sequence of the L-shaped block $b_{L}$. It should be noted that this operation will not pull other blocks up due to the DFS packing order induced by the $\mathrm{B}^{*}$-tree.

(2) Plateau: The contour is higher than the top profile sequence at the position of the current subblock $b_{2}$. (See Figure 7(b).) In this case, we pull $b_{1}$ up to conform to the top profile sequence of $b_{L}$. (Note that $b_{2}$ cannot be moved down because the compaction operation makes $b_{2}$ be placed right above another block.)

It is clear that each of the adjustments can be performed in constant time with the contour data structure.

In the following, we discuss the rotation and flip operations of an L-shaped block. For each L-shaped block $b_{i}$, there are eight orientations by rotation and 


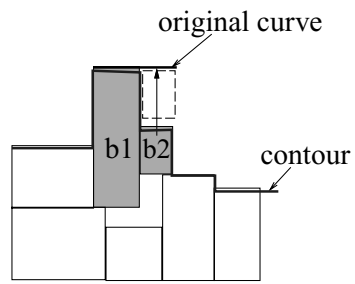

(a)

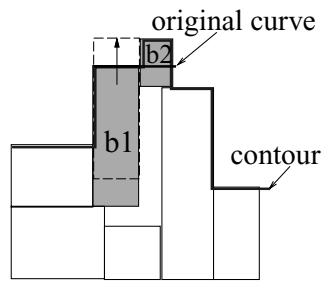

(b)

Fig. 7. Placing two subblocks $b_{1}$ and $b_{2}$ of an L-shaped block: (a) if the contour is lower than the top profile sequence at $b_{2}$, then we pull $b_{2}$ up to meet the top profile sequence; (b) if the contour is higher than the top profile sequence at $b_{2}$, then we pull $b_{1}$ up to meet the top profile sequence.

flip, as shown in Figure 4. To preserve the LC relation and keep it in the $\mathrm{B}^{*}$-tree, we repartition $b_{i}$ into two subblocks after it is rotated or flipped and keep the $L C$ relation between them. Figure 4 shows the subblocks after repartitioning. As shown in the figure, an L-shaped block is always partitioned by slicing it along the middle vertical boundary. After repartitioning, we should update the top profile sequence for the block.

\section{FLOORPLAN ALGORITHM}

Our rectilinear floorplan design algorithm is based on the simulated annealing method [Kirkpatrick et al. 1983; Sechen and Sangiovanni 1985] and the B*-tree described in Section 3 . We perturb a B*-tree (a feasible solution) to another B*tree by using the following four operations.

Op1: Rotate a block.

Op2: Flip a block.

Op3: Move a block to another place.

Op4: Swap two blocks.

The Op1 and Op2 operations have been described in Section 4. The Op3 operation deletes and inserts a block into a $\mathrm{B}^{*}$-tree. If the deleted node is associated with a rectangular block, we simply delete the node from the $\mathrm{B}^{*}$-tree. Otherwise, there will be two nodes associated with an L-shaped block, and we must delete the two nodes from the $\mathrm{B}^{*}$-tree and insert them in other places. Note that the $L C$ relations must hold. Both of the Op3 and Op4 operations need to apply the $\operatorname{Insert}\left(n_{i}\right)$ and $\operatorname{Delete}\left(n_{i}\right)$ operations, where $\operatorname{Insert}\left(n_{i}\right)\left(\operatorname{Delete}\left(n_{i}\right)\right)$ is the operation for inserting (deleting) a node $n_{i}$ to (from) a B*-tree. The $\mathrm{B}^{*}$-tree must remain a binary tree after deletion or insertion. We detail the deletion and insertion operations in the following.

\subsection{Deletion}

The deletion can be categorized into these cases:

-Case 1: A leaf node;

- Case 2: A node with one child;

- Case 3: A node with two children. 


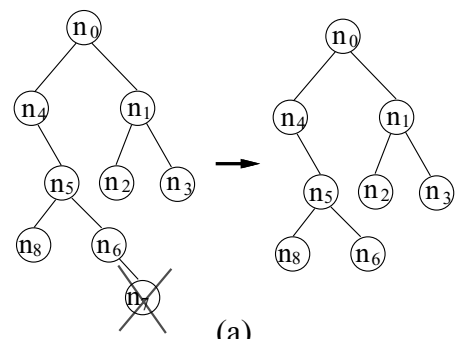

(a)

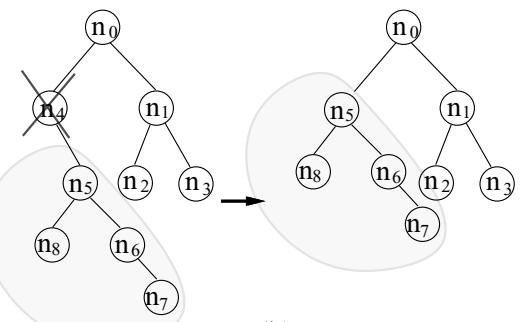

(b)

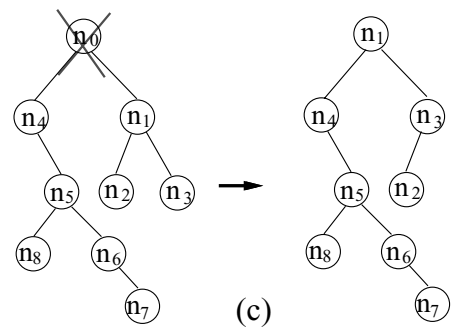

Fig. 8. Deletion: (a) deleting a leaf node; (b) deleting a node with only one child; (c) deleting a node with two children.

In Case 1, we can just delete the target leaf node directly, and the tree will still be a $\mathrm{B}^{*}$-tree. As shown in Figure 8(a), to delete the node $n_{7}$ from the $\mathrm{B}^{*}$-tree of Figure 2, we set the left child field of its parent $n_{6}$ to be NULL and free the node $n_{7}$.

In Case 2, we remove the target node and then place the single child at the position of the removed node. For example, after deleting the node $n_{4}$ from the $\mathrm{B}^{*}$-tree of Figure 2 , we move $n_{5}$ to the original position of $n_{4}$ and obtain the tree shown in Figure 8(b). This tree update can be performed in $O(1)$ time. Note that the relative positions of the blocks might be changed after the operation, and thus we might need to reconstruct a corresponding placement for further processing.

In Case 3 , when deleting a target node $n_{t}$ with two children, we replace $n_{t}$ by either its right child or left child $n_{c}$. Then we move a child of $n_{c}$ to the original position of $n_{c}$. The process proceeds until the corresponding leaf node is handled. For instance, suppose that we delete the node $n_{0}$ from the $\mathrm{B}^{*}$-tree of Figure 2. We can use the right child $n_{1}$ to replace it, and then use $n_{3}$ to replace $n_{1}$. (The resulting tree is shown in Figure 8(c).) It is obvious that such a deletion operation requires $O(h)$ time, where $h$ is the height of the $\mathrm{B}^{*}$-tree. Again the 


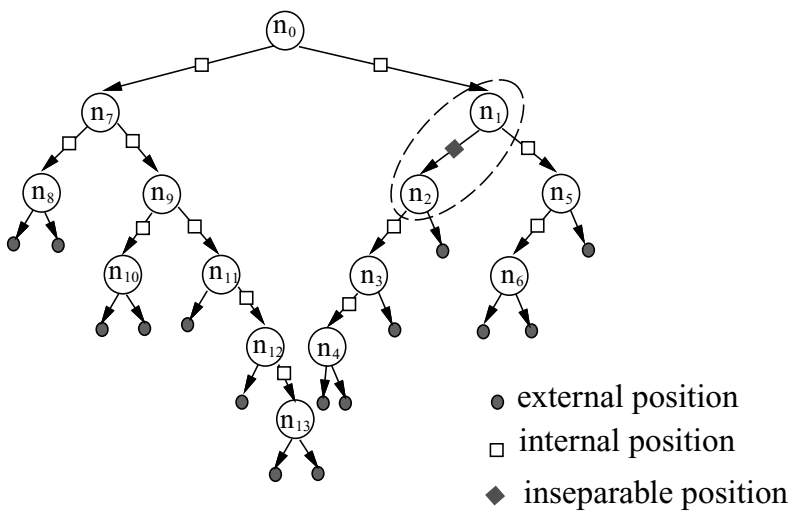

Fig. 9. The inseparable, internal, and external positions of a $\mathrm{B}^{*}$-tree. (Assume that $n_{1}$ and $n_{2}$ are associated with the same L-shaped block.) A node can be inserted at either an internal or an external position.

relative positions of the blocks might be changed after the operation, and thus we might need to reconstruct a corresponding placement for further processing.

Note that if the deleted node $n_{i}$ is a subblock of an L-shaped $b_{L}$, we should also delete the other subblock of $b_{L}$.

\subsection{Insertion}

When adding a block to a placement, we may place the block around a certain block, but not between two subblocks that belong to an L-shaped block. For a $B^{*}$-tree, we define three types of positions as follows. (See Figure 9 for an illustration.)

-Inseparable position: A position between two nodes associated with the two subblocks of an L-shaped block.

-Internal position: A position between two nodes in a $\mathrm{B}^{*}$-tree, but not an inseparable one.

-External position: A position pointed to by a NULL pointer.

Only internal and external positions can be used for inserting a new node.

For a rectangular block, we can insert it into an internal or an external position directly. For any L-shaped block $b_{L}$ consisting of two subblocks $b_{1}$ and $b_{2}$, with $b_{1}$ on the left-hand side of $b_{2}$, the two subblocks must be inserted into a $\mathrm{B}^{*}$-tree simultaneously, and $b_{2}$ must be the left child of $b_{1}$ (according to the $L C$ relation).

In the following, we discuss three cases of inserting an L-shaped block into an internal position. As shown in Figure 10, if we insert two nodes $b_{1}$ and $b_{2}$ of an L-shaped block into an internal position between nodes $b_{i}$ and $b_{j}$, with $b_{j}$ being a child of $b_{i}, b_{j}$ can be placed at the position that is the left child of $b_{2}$, the right child of $b_{2}$, or the right child of $b_{1}$. 


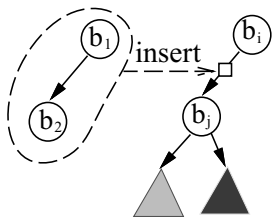

(a)

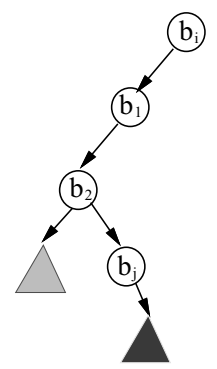

(c)

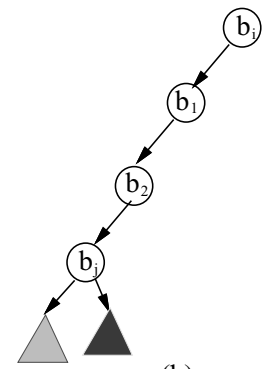

(b)

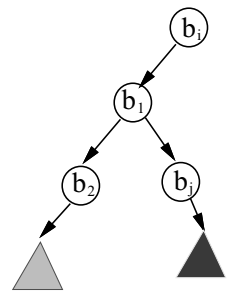

(d)

Fig. 10. Three cases of inserting an L-shaped block to an internal position.

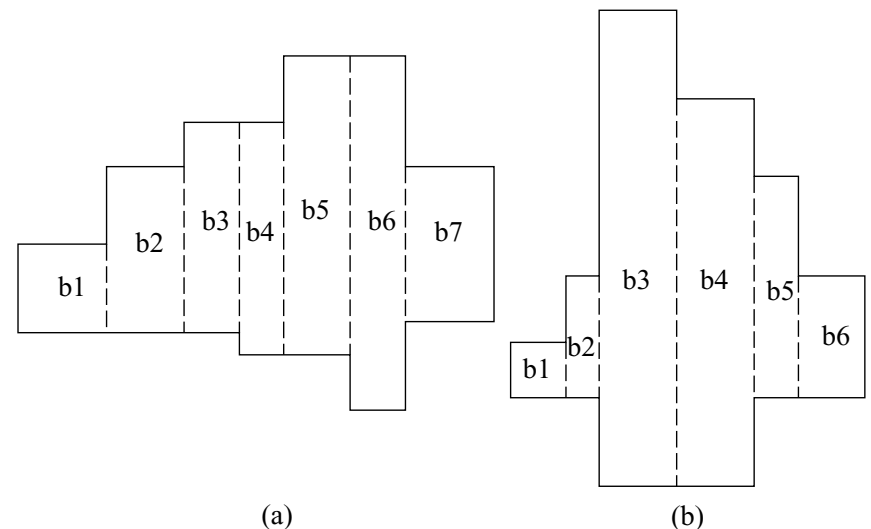

(a)

(b)

Fig. 11. (a) Partition a convex block along every vertical boundary from left to right; (b) repartition the block of (a) after it rotates.

\section{EXTENSION TO GENERAL RECTILINEAR BLOCKS}

In this section, we extend the techniques described in previous sections to handle general rectilinear blocks. In general, a rectilinear block can be partitioned into a set of rectangular subblocks. Let $b_{i}$ denote an arbitrarily shaped rectilinear block. $b_{i}$ can be partitioned into a set of rectangular subblocks by slicing $b_{i}$ from left to right along every vertical boundary of $b_{i}$, as shown in Figure 11(a).

After perturbing the Op1 and Op2 operations, we repartition a rectilinear block when it is rotated or flipped. Figure 11(b) shows the block of 


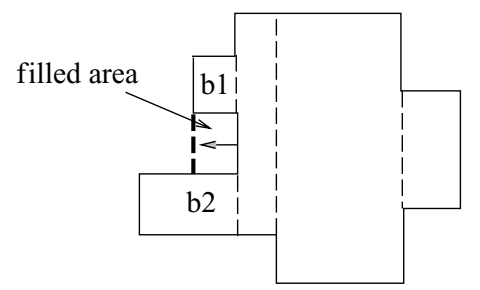

Fig. 12. Filling approximation for a rectilinear block.

Figure 11(a) after rotating $90^{\circ}$ clockwise; there are six subblocks in it after the repartition.

There are two types of rectilinear blocks: convex and concave. A rectilinear block is convex if any two points within the block can be connected by a shortest Manhattan path that also lies within the block; the block is concave, otherwise. Figures 11 and 12 show two convex and a concave block, respectively. A convex block $b_{C}$ can be partitioned into a set of subblocks $b_{1}, b_{2}, \ldots, b_{n}$ ordered from left to right. Considering the $L C$ relation, we keep the subblock $b_{i+1}$ as $b_{i}$ 's left child in the $\mathrm{B}^{*}$-tree to ensure that they are placed side by side along the $x$-direction, where $1 \leq i \leq n-1$. To ensure that $b_{1}, b_{2}, \ldots, b_{n}$ are not misaligned, we modify the processing for Basin and Plateau as follows.

-Basin: The contour is lower than the top profile sequence at the position of a subblock. We pull the subblock up to conform to the top profile sequence.

-Plateau: The top boundary of a subblock $b_{i}(1 \leq i \leq n)$ in the contour is higher than the top profile sequence at the position of $b_{i}$. Assume that $b_{i}$ has the largest top boundary. We pull all subblocks, except $b_{i}$, up to conform to the top profile sequence.

Moreover, all subblocks must be deleted (or inserted) together for the OP3 and OP4 operations.

For a concave block, there might be empty space between two subblocks. As shown in Figure 12, the subblock $b_{1}$ is placed above the subblock $b_{2}$, which cannot be characterized by an $L C$ relation in the $\mathrm{B}^{*}$-tree. Nevertheless, we can fill the concave holes of a concave block and make it a convex block. We call this operation a filling approximation for the rectilinear block. For any concave block, we treat it as a convex block after applying appropriate filling.

\section{EXPERIMENTAL RESULTS}

We implemented our algorithm in the $\mathrm{C}++$ programming language on a $450 \mathrm{MHz}$ SUN Ultra Sparc-I workstation with $1 \mathrm{~Gb}$ memory. Since the benchmarks in previous work are artificial cases and unavailable to us, we generate some general benchmarks for experiments in this article. Our test cases were generated by cutting a rectangle into a set of blocks. Therefore, the optimum area is given by the original block.

As shown in Table I, Columns 2 through 4 list the numbers of rectangular, L-shaped, and T-shaped blocks. RL10, RL20, and RL30 consist of only 
Table I. Experimental Results

\begin{tabular}{|l|c|c|c|c|c|c|c|}
\hline \hline Circuits & $\begin{array}{c}\text { \#Rectangular } \\
\text { Blocks }\end{array}$ & $\begin{array}{c}\text { \#L-Shaped } \\
\text { Blocks }\end{array}$ & $\begin{array}{c}\text { \#T-Shaped } \\
\text { Blocks }\end{array}$ & $\begin{array}{c}\text { Optimum } \\
\text { Area }\end{array}$ & $\begin{array}{c}\text { Resulting } \\
\text { Area }\end{array}$ & $\begin{array}{c}\text { Dead } \\
\text { Space }(\%)\end{array}$ & $\begin{array}{c}\text { Run-time } \\
(\mathrm{sec})\end{array}$ \\
\hline RL10 & 5 & 5 & 0 & $\begin{array}{c}100 \\
(10 \times 10)\end{array}$ & $\begin{array}{c}100 \\
(10 \times 10)\end{array}$ & 0.00 & 8 \\
\hline RL20 & 10 & 10 & 0 & $\begin{array}{c}400 \\
(20 \times 20)\end{array}$ & $\begin{array}{c}408 \\
(15 \times 27)\end{array}$ & 2.00 & 307 \\
\hline RL30 & 15 & 15 & 0 & $\begin{array}{c}900 \\
(30 \times 30)\end{array}$ & $\begin{array}{c}936 \\
(29 \times 32)\end{array}$ & 4.00 & 1636 \\
\hline RLT10 & 4 & 3 & 3 & $\begin{array}{c}100 \\
(10 \times 10)\end{array}$ & $\begin{array}{c}102 \\
(6 \times 17)\end{array}$ & 2.00 & 41 \\
\hline RLT20 & 7 & 7 & 6 & $\begin{array}{c}400 \\
(20 \times 20)\end{array}$ & $\begin{array}{c}414 \\
(18 \times 23)\end{array}$ & 3.50 & 1096 \\
\hline RLT30 & 10 & 10 & 10 & $\begin{array}{c}900 \\
(30 \times 30)\end{array}$ & $\begin{array}{c}945 \\
(27 \times 35)\end{array}$ & 5.00 & 3007 \\
\hline \hline
\end{tabular}

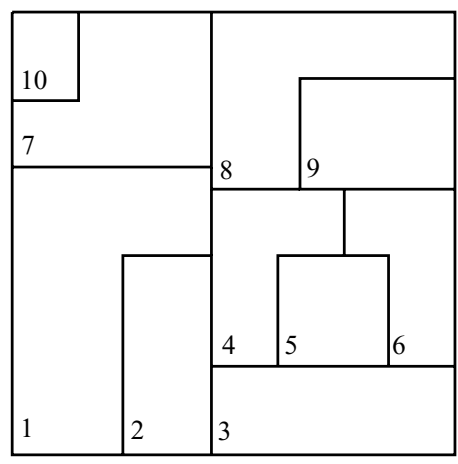

(a)

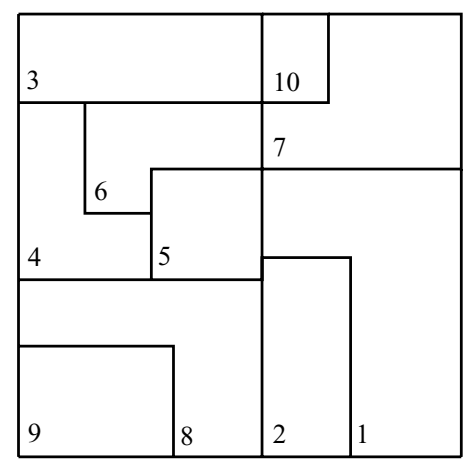

(b)

Fig. 13. Placement for RL10: 5 rectangular and 5 L-shaped blocks: (a) the optimum placement $(10 \times 10)$; $(b)$ the resulting placement $(10 \times 10)$.

rectangular and L-shaped blocks. There are 5 rectangular and 5 L-shaped blocks in RL10, 10 rectangular and 10 L-shaped blocks in RL20, and 15 rectangular and 15 L-shaped blocks in RL30, respectively. RLT10, RLT20, and RLT30 consist of not only rectangular and L-shaped blocks, but also T-shaped ones. RLT10 is composed of 4 rectangular, 3 L-shaped, and 3 T-shaped blocks; RLT20 is composed of 7 rectangular, 7 L-shaped, and 6 T-shaped blocks; and RLT30 is composed of 10 rectangular, $10 \mathrm{~L}$-shaped, and $10 \mathrm{~T}$-shaped blocks. The original area of each test case is shown in Column 5. Columns 6 and 7 list the resulting area and the dead space (\%). The results show that our algorithm obtains the optimum area for RL10 and near optimum areas for RL20, RL30, RLT10, RLT20, and RLT30 with areas only $2.00,4.00,2.00,3.50$, and $5.00 \%$ away from the optima, respectively. The run-times for achieving the results ranged from about 8 seconds to 50 minutes (see Column 8). Figures 13 and 14 show the optimum and the resulting placement for RL10 and RLT30, respectively. 


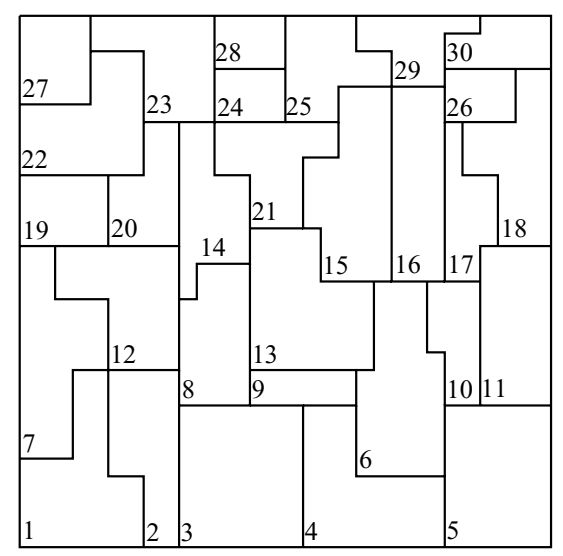

(a)

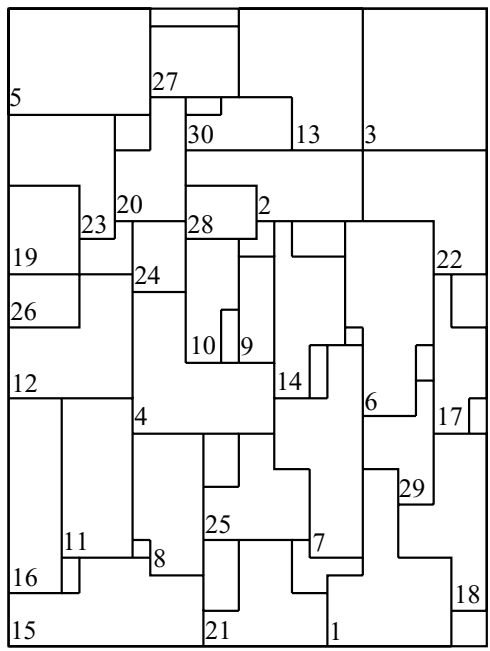

(b)

Fig. 14. Placement for RLT30: 10 rectangular, 10 L-shaped, and 10 T-shaped blocks: (a) the optimum placement $(30 \times 30)$; (b) the resulting placement $(27 \times 35)$.

\section{CONCLUSIONS}

In this article, we have extended the $\mathrm{B}^{*}$-tree approach introduced in Chang et al. [2000] to handle the placement of arbitrarily shaped rectilinear blocks. We partitioned a rectilinear block into a set of rectangular subblocks, each individually represented by a node in the $\mathrm{B}^{*}$-tree. The $L C$ relations and the basin and plateau operations were used to ensure that each block kept its original shape. The experiment results have shown that our approach is very effective in area utilization.

\section{REFERENCES}

Chang, Y.-C., Chang, Y.-W., Wu, G.-M., ANd Wu, S.-W. 2000. B*-Trees: A new representation for non-slicing floorplans. In Proceedings of the ACM/IEEE Design Automation Conference, (Los Angeles, June), 458-463.

Guo, P.-N., Cheng, C.-K., And Yoshimura, T. 1999. An O-tree representation of non-slicing floorplan and its applications. In Proceedings of the ACM/IEEE Design Automation Conference (California, June), 268-273.

Papadimitriou, C. and Steiglitz, K. 1982. Combinatorial Optimization:Algorithms and Complexity. Prentice-Hall Inc., Englewood Cliffs, NJ.

KANG, M. Z. AND DAI, W. 1997. General floorplanning with L-shaped, T-shaped and soft blocks based on bounded slicing grid structure. In Proceedings of the ACM / IEEE Asia and South Pacific Design Automation Conference (Chiba, Japan, January 28-31), 265-270.

KANG, M. Z. AND DAI, W. 1998. Arbitrary rectilinear block packing based on sequence pair. In Proceedings of the ACM/IEEE International Conference on Computer-Aided Design (San Jose, CA, November 8-12), 259-266.

Kirkpatrick, S., Gelatt, C. D., and Vecchi, M. P. $1983 . \quad$ Optimization by simulated annealing. Science 220, 4598, 671-680.

LEE, T. C. 1993. A bounded 2D contour searching algorithm for floorplan design with arbitrarily shaped rectilinear and soft modules. In Proceedings of the ACM/IEEE Design Automation Conference (California, June), 525-530. 
Murata, H. and Kun, E. S. 1998. Sequence pair based placement method for hard/soft/pre-placed modules. In Proceedings of the ACM International Symposium on Physical Design (California, April), 167-172.

Murata, H., FujiYoshi, K., NaKatake, S., and KajtTani, Y. 1995. Rectangle-packing based module placement. In Proceedings of the ACM / IEEE International Conference on Computer-Aided Design (California, November), 472-479.

Murata, H., Fujiyoshi, K., AND KaneKo, M. 1997. VLSI/PCB placement with obstacles based sequence pair. In Proceedings of the ACM International Symposium on Physical Design (California, April), 26-31.

Nakatake, S., Fujiyoshi, K., Murata, H., and Kajttani, Y. 1996. Module placement on BSGstructure and IC layout applications. In Proceedings of ACM/IEEE International Conference on Computer-Aided Design (California, November), 484-491.

Nakatake, S., FuruYa, M., AND KaJitani, Y. 1998. Module placement on BSG-structure with preplaced modules and rectilinear modules. In Proceedings of the ACM / IEEE Asia and South Pacific Design Automation Conference (Yokohama, February 10-13), 571-576.

Оттел, R. H. J. M. 1982. Automatic floorplan design. In Proceedings of the ACM/IEEE Design Automation Conference (California, June), 261-267.

Preas, B. T. And vanCleemput, W. M. 1979. Placement algorithms for arbitrarily shaped Blocks. In Proceedings of the ACM / IEEE Design Automation Conference (California, June), 474-480.

Sechen, C., and Sangiovanni-Vincentelli, A. 1985. The TimberWolf placement and routing package. IEEE J. Solid-State Circ. 20, 2 (April), 510-522.

WANG, T. C. AND Wong, D. F. 1990. An optimal algorithm for floorplan and area optimization. In Proceedings of the ACM/IEEE Design Automation Conference (Orlando, FL, June 24-28), 474-480.

Wong, D. F. AND LiU, C. L. 1986. A new algorithm for floorplan design. In Proceedings of the ACM / IEEE Design Automation Conference (California, June), 101-107.

Wong, D. F. AND LiU, C. L. 1987. Floorplan design for rectangular and L-shaped modules. In Proceedings of the IEEE / ACM International Conference on Computer-Aided Design (California, November), 520-523.

Xu, J., Guo, P.-N., ANd Cheng, C.-K. 1998. Rectilinear block placement using sequence-pair. In Proceedings of the ACM International Symposium on Physical Design (California, April), 173-178.

Received March 2001; accepted May 2002 